\title{
The effect of temperature and pressure on the self-assembly of dipalmitoylphosphatidylcholine using coarse-grained molecular dynamics
}

\begin{abstract}
The use of coarse-grained molecular dynamics (CG-MD) technique is a promising tool to explore the time and length scales of real physical system beyond what is feasible with atomistic molecular dynamics (MD). Here, structural and dynamics properties of the bilayer comprising 64 molecules of dipalmitoylphosphatidylcholine (DPPC) in water were investigated using coarse-grained molecular dynamics (CG-MD) simulation method. This was done to explore the effect of temperature and pressure on the self-assembly of DPPC. The models prepared were simulated at the temperatures of $298 \mathrm{~K}$ and $323 \mathrm{~K}$ under isotropic and semi-isotropic pressures. The aggregation started from the random configurations followed by forming bilayers in the period of $500 \mathrm{~ns}$ for all model systems. Area per lipid, thickness, and radial distribution values were different for each model while the bilayer formation pattern was the same.
\end{abstract}

Keyword: Dipalmitoylphosphatidylcholine; Self-assembly; Coarse-grained molecular dynamics 